\title{
Competition between disorder and interaction effects in three-dimensional Weyl semimetals
}

\author{
J. González \\ Instituto de Estructura de la Materia, Consejo Superior de Investigaciones Científicas, Serrano 123, 28006 Madrid, Spain
}

(Received 10 April 2017; revised manuscript received 19 June 2017; published 9 August 2017)

\begin{abstract}
We investigate the low-energy scaling behavior of an interacting three-dimensional (3D) Weyl semimetal in the presence of disorder. In order to achieve a renormalization group analysis of the theory, we first focus on the effects of a short-ranged-correlated disorder potential, checking nevertheless that this choice is not essential to locate the different phases of the Weyl semimetal. We show that there is a line of fixed points in the renormalization group flow of the interacting theory, corresponding to the disorder-driven transition to a diffusive metal phase. Along that boundary, the critical disorder strength undergoes a strong increase with respect to the noninteracting theory, as a consequence of the unconventional screening of the Coulomb and disorder-induced interactions. The complementary resolution of the Schwinger-Dyson equations allows us to determine the full phase diagram of the system, showing the prevalence of a renormalized semimetallic phase in the regime of intermediate interaction strength, and adjacent to the non-Fermi liquid instability of 3D Weyl semimetals in the strong interaction regime.
\end{abstract}

DOI: 10.1103/PhysRevB.96.081104

Introduction. During more than a decade we have witnessed the discovery of a number of materials whose electronic properties have been defeating the conventional description of solid state systems. Starting with graphene [1] and ending with the three-dimensional (3D) Weyl semimetals [2,3], these materials display degenerate bands that touch only at isolated points in momentum space, with a linear dispersion similar to that of relativistic particles. This introduces novel topological features in the description of the electron systems [4-16], which has important consequences for the transport properties of the materials.

The Coulomb interaction has to play also an important role in those systems, as it remains long ranged at the nodal points. A key property is the scale dependence of the quasiparticle parameters at low energies [17], already observed in the case of the Fermi velocity of graphene [18]. Regarding the 3D semimetals, similar effects have to exist implying the renormalization of the Fermi velocity [19,20], as well as of the electron quasiparticle weight. It has been shown that, for sufficiently large number of nodal points, the renormalization of the latter should be the dominant effect at low energies, with the potential to drive the system to a non-Fermi liquid phase $[21,22]$.

In this picture, it would be convenient to assess the impact of disorder in the electron system. It was found long ago that, in the presence of a random disorder potential, 3D semimetals may undergo a transition to a phase characterized by developing a nonvanishing density of states at the nodal points [23]. Recently, there has been much interest in understanding the nature of that transition [24-42]. It remains unknown to a large extent, however, whether the disorder can modulate the interaction effects in such semimetals or, vice versa, whether the long-range Coulomb interaction can modify the disorder-driven transition (see nevertheless Ref. [43]).

In this Rapid Communication, we investigate the lowenergy scaling behavior of an interacting 3D Weyl semimetal when it is under the effect of a random disorder potential. We show that the most important feature is the unconventional screening of the Coulomb and the disorder-induced interactions, implying a strong increase of the critical disorder strength for the disorder-driven transition. We will see that this has a reflection in the phase diagram of the system, leading to the prevalence of a renormalized semimetallic phase in the whole regime of intermediate interaction strength, and adjacent to the non-Fermi liquid instability characteristic of the strong interaction regime of 3D Weyl semimetals.

Large- $N$ renormalization group approach. Our starting point is a model of $2 N$ two-component Weyl spinors $\left\{\psi_{i}\right\}$ with long-range Coulomb interaction, governed by the action

$$
\begin{aligned}
S= & \int d^{3} r d t \psi_{i}^{\dagger}(\mathbf{r})\left(-i \partial_{t}-i v_{F} \gamma_{0} \boldsymbol{\gamma} \cdot \boldsymbol{\partial}-e_{0} \phi(\mathbf{r})\right) \psi_{i}(\mathbf{r}) \\
& +\int d^{3} r \psi_{i}^{\dagger}(\mathbf{r}) \psi_{i}(\mathbf{r}) \eta(\mathbf{r}),
\end{aligned}
$$

where $\eta(\mathbf{r})$ is the field representing the disorder, $\phi(\mathbf{r})$ stands for the scalar interaction potential, and $\left\{\gamma_{\alpha}\right\}$ is a set of matrices satisfying $\left\{\gamma_{\alpha}, \gamma_{\beta}\right\}=2 g_{\alpha \beta}$ [44]. Assuming that the Fermi velocity $v_{F}$ is typically much smaller than the speed of light, we can neglect retardation effects in the $e-e$ interaction and take the free propagator of the scalar potential in momentum space as $D_{0}(\mathbf{q}, \omega)=1 / \mathbf{q}^{2}$. On the other hand, $\eta(\mathbf{r})$ corresponds in general to a random potential with zero average and a variance

$$
\overline{\eta(\mathbf{r}) \eta\left(\mathbf{r}^{\prime}\right)}=w\left(\mathbf{r}-\mathbf{r}^{\prime}\right) .
$$

For the sake of dealing with an action $S$ which is scale invariant, we will focus in this section on the effects of short-ranged correlated disorder, adopting a distribution $w(\mathbf{r})=w_{0} / \mathbf{r}^{2}$.

We consider the case of quenched disorder, which can be treated using the replica method. This leads us to introduce a number $n$ of different copies of the fields $\phi$ and $\psi_{i}$ in the action (1), interacting with the random potential $\eta(\mathbf{r})$. Those fields get then an additional index $a$ running over the $n$ copies, with the need to take the limit $n \rightarrow 0$ at the end of every calculation in order to obtain physical quantities.

The replica method can be applied to the computation of the electron self-energy in the large- $N$ limit. On the one hand, there is the RPA contribution of the disorder-free theory, already considered in Ref. [21]. On the other hand, the disorder potential introduces a new contribution, which is given in the large- $N$ limit by the sum of rainbow diagrams of the type shown in Fig. 1. Note that, in this approximation 


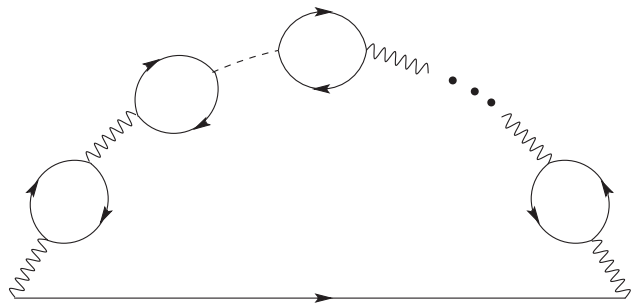

FIG. 1. Class of self-energy diagrams correcting the fermion propagator (represented by a directed line) to leading order in the large- $N$ limit, with multiple interactions mediated by the Coulomb potential (wavy line) and constrained to a single correlation of the disorder potential (dashed line) in order to render a nonvanishing contribution in the limit of number of replicas $n \rightarrow 0$.

(which amounts also to neglect vertex corrections), the sum is restricted to diagrams with just a single correlation $w(\mathbf{r})$, since the inclusion of more propagators of the random potential makes the corresponding rainbow diagram vanish in the limit $n \rightarrow 0$.

The sum of the diagrams of the type shown in Fig. 1 leads to a contribution to the electron self-energy [45]

$$
\Sigma^{(w)}(\mathbf{k}, \omega)=\frac{w_{0}}{e_{0}^{4}} \int \frac{d^{3} p}{(2 \pi)^{3}} \frac{2 \pi^{2}}{|\mathbf{p}|} \frac{G_{0}(\mathbf{k}-\mathbf{p}, \omega)}{\left(\frac{1}{e_{0}^{2}}+\frac{N}{6 \pi^{2} v_{F}} \log \frac{\Lambda}{|\mathbf{p}|}\right)^{2}},
$$

where $G_{0}(\mathbf{k}, \omega)$ stands for the free fermion propagator and $\Lambda$ is a high-momentum cutoff. This has to be reabsorbed into a suitable redefinition of the parameters of the model to obtain meaningful physical results. Interestingly, that can be achieved by means of the same renormalization demanded by the disorder-free interacting theory, that is, by introducing the renormalized charge $e$ through the relation $1 / e^{2}=1 / e_{0}^{2}+$ $\left(N / 6 \pi^{2} v_{F}\right) \log (\Lambda / \mu)$. To guarantee the cutoff independence of (3), one still has to impose that the prefactor $w_{0} / e_{0}^{4}$ does not depend on $\Lambda$, which can be done by introducing a renormalized disorder strength $w_{R}$ such that $w_{R} / e^{4}=w_{0} / e_{0}^{4}$. Overall this implies the scaling of the couplings

$$
e^{2}(\mu)=\frac{e_{0}^{2}}{1+\frac{N e_{0}^{2}}{6 \pi^{2} v_{F}} \log \frac{\Lambda}{\mu}}, \quad w_{R}(\mu)=\frac{w_{0}}{\left(1+\frac{N e_{0}^{2}}{6 \pi^{2} v_{F}} \log \frac{\Lambda}{\mu}\right)^{2}} .
$$

The last expression in (4) is the reflection that the disorder we are dealing with is a marginally irrelevant perturbation of the interacting theory in the low-energy limit $\mu \rightarrow 0$.

The fact that the renormalized strength $w_{R}$ scales to zero at low energies does not mean however that there cannot be interesting effects driven by the disorder. In the present approach, those effects arise from the scaling of the electron quasiparticle parameters. The dressed fermion propagator $G(\mathbf{k}, \omega)$ has to be independent of the high-momentum cutoff $\Lambda$, which requires one to introduce renormalization factors $Z_{\psi}$ for the quasiparticle weight and $Z_{v}$ for the Fermi velocity in the expression

$$
G(\mathbf{k}, \omega)^{-1}=Z_{\psi}\left(\omega-Z_{v} v_{R} \gamma_{0} \gamma \cdot \mathbf{k}\right)-Z_{\psi} \Sigma(\mathbf{k}, \omega) .
$$

In this last equation, $\Sigma$ stands for the sum of the disorder-free and the $\Sigma^{(w)}$ contribution. This latter becomes particularly

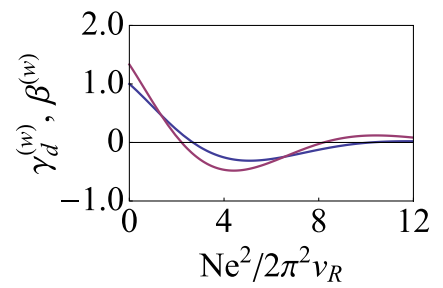

(a)

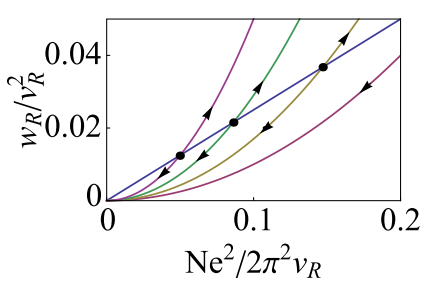

(b)
FIG. 2. (a) Plot of the contribution from disorder to the electron anomalous dimension $\gamma_{d}$ (blue line) and to the rate of variation of the Fermi velocity $\beta$ (red line) in the large- $N$ approximation. (b) Renormalization group flow in the low-energy limit of the effective strengths of the disorder and the Coulomb interaction, obtained from the resolution of Eqs. (8) and (9). The blue line represents the set of unstable fixed points at $w_{R} / v_{R}^{2}=g / 4$.

important since it may lead to a significant reduction of the Fermi velocity, as we see in what follows.

In order to compute $Z_{\psi}$ and $Z_{v}$ up to high orders in $e^{2}$, it is convenient to turn to a dimensional regularization, instead of using a high-momentum cutoff. With that procedure, the momentum integrals are computed in dimension $D=3-\epsilon$, in such a way that the powers of $\log (\Lambda)$ are traded by poles in the $\epsilon$ parameter [45]. In terms of the effective coupling $g=N e^{2} / 2 \pi^{2} v_{R}$, the renormalization factors have in general the pole structure $Z_{\psi}=1+(1 / N) \sum_{n=1}^{\infty} c_{n}(g) / \epsilon^{n}, Z_{v}=1+$ $(1 / N) \sum_{n=1}^{\infty} b_{n}(g) / \epsilon^{n}$. The disorder-free contribution to the coefficients $c_{n}$ and $b_{n}$ has been already studied in Ref. [21]. We report here the results from switching on the effects of disorder represented by the self-energy contribution $\Sigma^{(w)}$.

We note that the knowledge of $Z_{\psi}$ allows one to compute the electron anomalous dimension $\gamma_{d}$ from the dependence of the renormalized theory on the auxiliary scale $\mu$, which gives $\gamma_{d}(g)=\left(\mu / Z_{\psi}\right)\left(\partial Z_{\psi} / \partial \mu\right)$ [46]. Moreover, one can also exploit the fact that the unrenormalized theory does not know about $\mu$ to enforce the independence of the bare Fermi velocity on that auxiliary scale, expressed as $\partial\left(Z_{v} v_{R}\right) / \partial \mu=0$. This leads to a scaling equation for the renormalized Fermi velocity

$$
\frac{\mu}{v_{R}} \frac{\partial v_{R}}{\partial \mu}=\beta(g)
$$

In practice, one takes advantage of the fact that only the first residues $c_{1}$ and $b_{1}$ contribute to $\gamma_{d}$ and $\beta$ [45]. We have computed in particular the part linear in $w_{R}$ of the residues $c_{1}$ and $b_{1}$ up to very large orders in the effective interaction strength $g=N e^{2} / 2 \pi^{2} v_{R}$. These expansions, together with those in Ref. [21], allow us to construct the electron anomalous dimension as $\gamma_{d}=(1 / N) \gamma_{d}^{(0)}(g)+\left(w_{R} / v_{R}^{2}\right) \gamma_{d}^{(w)}(g)+O\left(w_{R}^{2}\right)$ and the scaling of the Fermi velocity as $\beta=(1 / N) \beta^{(0)}(g)+$ $\left(w_{R} / v_{R}^{2}\right) \beta^{(w)}(g)+O\left(w_{R}^{2}\right)$. The results obtained for $\gamma_{d}^{(w)}(g)$ and $\beta^{(w)}(g)$, valid up to values of $g$ deep into the strongcoupling regime, are plotted in Fig. 2(a).

It is important to stress that $\gamma_{d}^{(w)}(g)$ does not show any singular behavior in the range of couplings covered in Fig. 2(a). One of the main results reported in Ref. [21] was that the disorder-free contribution $\gamma_{d}^{(0)}(g)$ diverges at a critical value of the effective coupling $g_{c}=3$. We may conclude therefore that the disorder effects here analyzed do not prevent 
the development of the non-Fermi liquid phase characteristic of the strong-interaction regime of Weyl semimetals [21,22].

On the other hand, $\beta^{(w)}$ leads to a positive contribution to the right-hand side of Eq. (6), which reads to lowest order in the couplings [45]

$$
\frac{\mu}{v_{R}} \frac{\partial v_{R}}{\partial \mu}=-\frac{1}{6 \pi^{2}} \frac{e^{2}}{v_{R}}+\frac{4}{3} \frac{w_{R}}{v_{R}^{2}}+\cdots .
$$

This equation can be used to find the low-energy behavior of the effective strengths $g=N e^{2} / 2 \pi^{2} v_{R}$ and $w_{R} / v_{R}^{2}$. The scaling in Eq. (4) can be encoded in the two equations $\mu(\partial / \partial \mu) e^{2}=N e^{4} / 6 \pi^{2} v_{R}$ and $\mu(\partial / \partial \mu) w_{R}=$ $N w_{R} e^{2} / 3 \pi^{2} v_{R}$. Then, we get to quadratic order in the couplings (keeping dominant terms in the large- $N$ limit)

$$
\begin{gathered}
\mu \frac{\partial}{\partial \mu} g=\frac{1}{3} g^{2}-\frac{4}{3} \frac{w_{R}}{v_{R}^{2}} g+\cdots, \\
\mu \frac{\partial}{\partial \mu} \frac{w_{R}}{v_{R}^{2}}=\frac{2}{3} \frac{w_{R}}{v_{R}^{2}} g-\frac{8}{3}\left(\frac{w_{R}}{v_{R}^{2}}\right)^{2}+\cdots .
\end{gathered}
$$

Quite remarkably, Eqs. (8) and (9) reveal the existence of a line of unstable fixed points at $w_{R} / v_{R}^{2}=g / 4$, as shown in Fig. 2(b). Below that line, the theory scales in the low-energy limit $\mu \rightarrow 0$ towards the noninteracting regime. Above the critical line, the effective couplings flow away from the weak-coupling regime as a manifestation of the dominant effects of disorder, signaling the onset of a phase whose precise characterization requires a more comprehensive nonperturbative approach.

Schwinger-Dyson equations. To get more information about the phase induced by disorder, we resort to a self-consistent resolution of the Schwinger-Dyson equations of the electron system. In this approach, we are going to adopt a truncation of the equations that amounts to include all kind of diagrammatic contributions except those containing vertex corrections. Then, the electron propagator $G(\mathbf{k}, \omega)$ is bound to satisfy the equation

$$
i \Sigma(\mathbf{k}, \omega)=-\int \frac{d^{3} p}{(2 \pi)^{3}} \frac{d \omega_{p}}{2 \pi} G\left(\mathbf{k}-\mathbf{p}, \omega-\omega_{p}\right) D\left(\mathbf{p}, \omega_{p}\right),
$$

where $D\left(\mathbf{p}, \omega_{p}\right)$ stands for the dressed interaction propagator. In the bare vertex approximation, that interaction includes the RPA sum of diagrams of the disorder-free theory plus a similar sum with just a single correlation of the disorder potential replacing each time one of the Coulomb potentials (in analogy with the large- $N$ diagrams in Fig. 1). The difference with respect to the previous large- $N$ approach is that now the electron-hole polarization $\Pi\left(\mathbf{q}, \omega_{q}\right)$ must be computed in terms of the dressed electron propagator according to the expression

$$
i \Pi\left(\mathbf{q}, \omega_{q}\right)=-\int \frac{d^{3} p}{(2 \pi)^{3}} \frac{d \omega_{p}}{2 \pi} \operatorname{Tr}\left[G\left(\mathbf{p}+\mathbf{q}, \omega_{p}+\omega_{q}\right) G\left(\mathbf{p}, \omega_{p}\right)\right] .
$$

It can be shown that the couple of equations (10) and (11) can be solved self-consistently by introducing the ansatz

$$
G(\mathbf{k}, \omega)=\left[z_{\psi}(\mathbf{k}, \omega)\left(\omega-z_{v}(\mathbf{k}, \omega) v_{F} \gamma_{0} \boldsymbol{\gamma} \cdot \mathbf{k}\right)\right]^{-1} .
$$

In practice, one may perform a numerical resolution of the integral equations by rotating all the frequencies in the complex

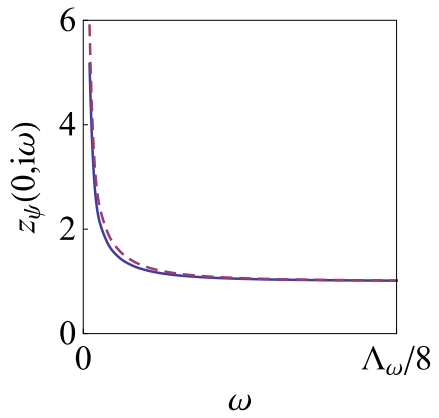

(a)

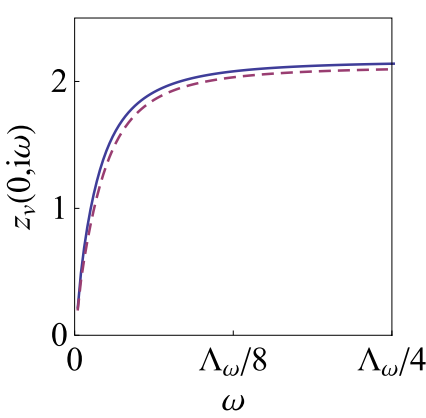

(b)
FIG. 3. Renormalization factors $z_{\psi}$ (a) and $z_{v}$ (b) from the selfconsistent resolution of the Schwinger-Dyson equations (with $N=$ 6 ). The full lines correspond to the coupling $w_{0} / v_{B}^{2} \approx 17.0$ on the transition driven by correlated disorder (blue line) in the diagram of Fig. 4(a), and the dashed lines to the coupling $w_{0} / v_{B}^{2} \approx 13.0 \mathrm{~nm}$ on the transition driven by uncorrelated disorder (blue line) in the diagram of Fig. 4(b).

plane, $\omega=i \bar{\omega}$, and working (in the case of the fermion propagator) with the discrete set $\bar{\omega}_{n}=(2 n+1) \pi k_{B} T$ running over integer numbers $n$. This amounts to place the theory at a finite temperature $T$. Furthermore, one has to cut off the integrals at a maximum value of the modulus of the momentum $\Lambda$ [47]. This momentum cutoff can be used then to assign the microscopic unrenormalized values of the physical parameters, like the bare Fermi velocity [as $v_{B}=z_{v}(\Lambda, 0) v_{F}$ ] or the square of the bare electron charge [as $\left.e_{B}^{2}=\Lambda^{2} D(\Lambda, 0)\right]$.

In the case of short-ranged correlated disorder with $w(\mathbf{r})=$ $w_{0} / \mathbf{r}^{2}$, the resolution of the Schwinger-Dyson equations shows indeed that the model has two different phases (apart from the non-Fermi liquid phase at strong interaction identified in Ref. [21]) depending on the values of $w_{0} / v_{B}^{2}$ and $\lambda=$ $e_{B}^{2} / 2 \pi^{2} v_{B}$. It turns out that there is a critical line, in the regime of small $\lambda$, separating a phase with regular behavior of $z_{\psi}$ and $z_{v}$ from a different phase whose onset is characterized by the divergence of $z_{\psi}$ and concomitant vanishing of $z_{v}$ in the limit $\bar{\omega} \rightarrow 0$. This is represented in Fig. 3, which shows a plot of those functions when the couplings reach the critical line. The complete phase diagram in the plane defined by the bare coupling strengths $w_{0} / v_{B}^{2}$ and $\lambda=e_{B}^{2} / 2 \pi^{2} v_{B}$ can be seen in Fig. 4(a) (for the model with $N=6$ ).

We can establish at this point the comparison with a similar resolution of the Schwinger-Dyson equations in the case of uncorrelated disorder with distribution $w(\mathbf{r})=w_{0} \delta(\mathbf{r})$. There appears then an analogous critical line mapping the disorderdriven transition, which is characterized again by the vanishing of the quasiparticle weight and the effective Fermi velocity, as seen in Fig. 3. The corresponding phase diagram is represented in Fig. 4(b). The only qualitative difference with respect to the diagram shown for correlated disorder is that the critical line for the disorder-driven transition tends to reach the origin in the diagram of Fig. 4(a) as $T \rightarrow 0$. This does not happen in the case of uncorrelated disorder [45], which is in agreement with a nonvanishing critical strength for such a type of disorder in the zero-temperature noninteracting theory.

The present approach also allows us to uncover the physical meaning of the phase above the disorder-driven transition. The 


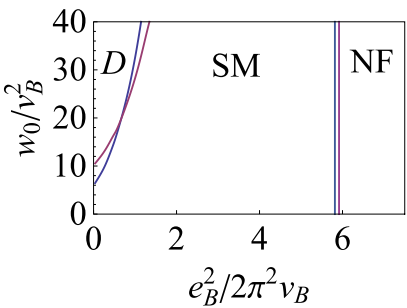

(a)

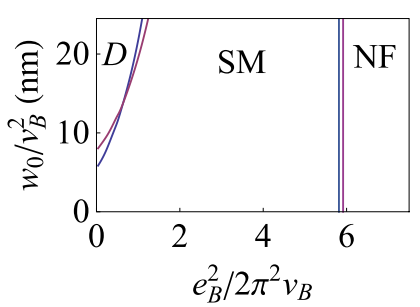

(b)
FIG. 4. Phase diagrams of the interacting 3D Weyl semimetal (for $N=6$ ) with (a) short-ranged-correlated and (b) uncorrelated disorder, showing the critical boundaries at $k_{B} T \approx 32 \mathrm{meV}$ (red lines) and $k_{B} T \approx 16 \mathrm{meV}$ (blue lines) which separate phases corresponding to a diffusive metal (D), semimetallic behavior (SM), and non-Fermi liquid behavior (NF).

expression of the propagator (12) can be applied to compute the density of states $n(\omega)$ at the nodal points as

$$
\begin{aligned}
n(0) & =\lim _{\omega \rightarrow 0} \operatorname{Im} \operatorname{Tr} \int d^{3} k G(\mathbf{k}, \omega) \\
& \sim \lim _{\omega \rightarrow 0} \operatorname{Im} \operatorname{Tr} \int d^{3} k \frac{1}{z_{\psi} z_{v}^{3}} G_{0}(\mathbf{k}, \omega) \sim \lim _{\omega \rightarrow 0} \frac{\omega^{2}}{z_{\psi} z_{v}^{3}} .
\end{aligned}
$$

With this estimate, one can check that the vanishing of $z_{v}(\mathbf{k}, 0)$ at the onset of the phase with dominant disorder corresponds to the appearance of a nonvanishing (not exponentially small) density of states in the limit $\omega \rightarrow 0$. We see therefore that the phase placed to the left in the diagrams of Figs. 4(a) and 4(b) is similar to the diffusive metal phase induced by disorder in the noninteracting 3D semimetals [23-34], which is promoted here to a narrow region in the phase diagram of the interacting theory.

Conclusion. We have seen that disorder and interaction effects tend to compete in 3D Weyl semimetals, converting the diffusive metal regime into a very narrow phase which reflects the irrelevance of the disorder effects in the presence of a sufficiently strong Coulomb interaction. We have seen that a semimetallic phase with renormalized parameters prevails in a large region of the phase diagram, extending over the whole regime of intermediate interaction strength and being adjacent to the non-Fermi liquid instability of 3D Weyl semimetals in the strong interaction regime.

In our analysis, the long-range component of the Coulomb interaction plays the dominant role, since short-range interactions are in general irrelevant in the low-energy limit of the 3D semimetals. Nevertheless, a strong short-range interaction can still lead to a broken-symmetry phase of the system in the regime where the long-range interaction does not give rise itself to a strong-coupling instability of the Weyl semimetal. According to the results of Refs. [21,22], this can only happen for a number of pairs of Weyl nodes $N \leqslant 4$. In that case, short-range interactions may couple Weyl nodes with different chiralities leading to the interplay between symmetry breaking and the disorder-driven effects [43]. In this regard, the conclusions reached in our study can be fully applied to Weyl semimetals in the range $N \geqslant 6$, which covers instances like TaAs, TaP, NbAs, as well as the family of the pyrochlore iridates.

The main conclusion of our work is that the effects of disorder can be in general disregarded in real 3D semimetals, due to the renormalization induced by the Coulomb interaction. For typical 3D semimetals with Fermi velocity $v_{F} \lesssim 1 \mathrm{eV} \mathrm{nm}$, the effective coupling $\lambda=e_{B}^{2} / 2 \pi^{2} v_{B}$ gets values of order $\gtrsim 1$. This means that these systems should naturally fall in the regimes with intermediate or strong interaction strength, displaying semimetallic or non-Fermi liquid behavior, but away from the diffusive metal phase confined by the steep disorder-driven transition shown in Fig. 4.

Acknowledgment. We acknowledge financial support from MINECO (Spain) through Grant No. FIS2014-57432-P.
[1] K. S. Novoselov, A. K. Geim, S. V. Morozov, D. Jiang, Y. Zhang, S. V. Dubonos, I. V. Grigorieva, and A. A. Firsov, Science 306, 666 (2004).

[2] S.-Y. Xu, I. Belopolski, N. Alidoust, M. Neupane, G. Bian, C. Zhang, R. Sankar, G. Chang, Z. Yuan, C.-C. Lee, S.-M. Huang, H. Zheng, J. Ma, D. S. Sanchez, B. Wang, A. Bansil, F. Chou, P. P. Shibayev, H. Lin, S. Jia, and M. Z. Hasan, Science 349, 613 (2015)

[3] B. Q. Lv, H. M. Weng, B. B. Fu, X. P. Wang, H. Miao, J. Ma, P. Richard, X. C. Huang, L. X. Zhao, G. F. Chen, Z. Fang, X. Dai, T. Qian, and H. Ding, Phys. Rev. X 5, 031013 (2015).

[4] K. Fukushima, D. E. Kharzeev, and H. J. Warringa, Phys. Rev. D 78, 074033 (2008).

[5] M. M. Vazifeh and M. Franz, Phys. Rev. Lett. 111, 027201 (2013).

[6] Y. Chen, S. Wu, and A. A. Burkov, Phys. Rev. B 88, 125105 (2013).

[7] D. T. Son and B. Z. Spivak, Phys. Rev. B 88, 104412 (2013).

[8] A. A. Burkov, Phys. Rev. B 91, 245157 (2015).
[9] J. Xiong, S. K. Kushwaha, T. Liang, J. W. Krizan, M. Hirschberger, W. Wang, R. J. Cava, and N. P. Ong, Science 350, 413 (2015).

[10] X. Wan, A. M. Turner, A. Vishwanath, and S. Y. Savrasov, Phys. Rev. B 83, 205101 (2011).

[11] K.-Y. Yang, Y.-M. Lu, and Y. Ran, Phys. Rev. B 84, 075129 (2011).

[12] A. A. Burkov and L. Balents, Phys. Rev. Lett. 107, 127205 (2011).

[13] G. Xu, H. Weng, Z. Wang, X. Dai, and Z. Fang, Phys. Rev. Lett. 107, 186806 (2011).

[14] W. Witczak-Krempa and Y. B. Kim, Phys. Rev. B 85, 045124 (2012).

[15] H. Weng, C. Fang, Z. Fang, B. A. Bernevig, and X. Dai, Phys. Rev. X 5, 011029 (2015).

[16] S.-M. Huang, S.-Y. Xu, I. Belopolski, C.-C. Lee, G. Chang, B. Wang, N. Alidoust, G. Bian, M. Neupane, C. Zhang, S. Jia, A. Bansil, H. Lin, and M. Z. Hasan, Nat. Commun. 6, 7373 (2015). 
[17] J. González, F. Guinea, and M. A. H. Vozmediano, Nucl. Phys. B 424, 595 (1994); Phys. Rev. B 59, R2474 (1999).

[18] D. C. Elias, R. V. Gorbachev, A. S. Mayorov, S. V. Morozov, A. A. Zhukov, P. Blake, L. A. Ponomarenko, I. V. Grigorieva, K. S. Novoselov, F. Guinea, and A. K. Geim, Nat. Phys. 7, 701 (2011).

[19] P. Hosur, S. A. Parameswaran, and A. Vishwanath, Phys. Rev. Lett. 108, 046602 (2012).

[20] B. Rosenstein and M. Lewkowicz, Phys. Rev. B 88, 045108 (2013).

[21] J. González, Phys. Rev. B 90, 121107(R) (2014); 92, 125115 (2015).

[22] J. González, J. High Energy Phys. (2015) 190.

[23] E. Fradkin, Phys. Rev. B 33, 3257 (1986); 33, 3263 (1986).

[24] R. Shindou and S. Murakami, Phys. Rev. B 79, 045321 (2009).

[25] P. Goswami and S. Chakravarty, Phys. Rev. Lett. 107, 196803 (2011).

[26] S. Ryu and K. Nomura, Phys. Rev. B 85, 155138 (2012).

[27] K. Kobayashi, T. Ohtsuki, K.-I. Imura, and I. F. Herbut, Phys. Rev. Lett. 112, 016402 (2014).

[28] B. Sbierski, G. Pohl, E. J. Bergholtz, and P. W. Brouwer, Phys. Rev. Lett. 113, 026602 (2014).

[29] Y. Ominato and M. Koshino, Phys. Rev. B 89, 054202 (2014).

[30] R. Nandkishore, D. A. Huse, and S. L. Sondhi, Phys. Rev. B 89, 245110 (2014).

[31] S. V. Syzranov, L. Radzihovsky, and V. Gurarie, Phys. Rev. Lett. 114, 166601 (2015).

[32] J. H. Pixley, P. Goswami, and S. D. Sarma, Phys. Rev. Lett. 115, 076601 (2015).

[33] C.-Z. Chen, J. Song, H. Jiang, Q.-f. Sun, Z. Wang, and X. C. Xie, Phys. Rev. Lett. 115, 246603 (2015).
[34] J. H. Pixley, D. A. Huse, and S. D. Sarma, Phys. Rev. X 6, 021042 (2016).

[35] S. Bera, J. D. Sau, and B. Roy, Phys. Rev. B 93, 201302(R) (2016).

[36] J. H. Pixley, D. A. Huse, and S. D. Sarma, Phys. Rev. B 94, 121107(R) (2016).

[37] T. Louvet, D. Carpentier, and A. A. Fedorenko, Phys. Rev. B 94, 220201(R) (2016).

[38] B. Roy, V. Juricic, and S. D. Sarma, Sci. Rep. 6, 32446 (2016).

[39] S. V. Syzranov, V. Gurarie, and L. Radzihovsky, Ann. Phys. (NY) 373, 694 (2016).

[40] T. Louvet, D. Carpentier, and A. A. Fedorenko, Phys. Rev. B 95, 014204 (2017).

[41] B. Roy, R.-J. Slager, and V. Juricic, arXiv:1610.08973.

[42] Y. Wu, H. Liu, H. Jiang, and X. C. Xie, Phys. Rev. B 96, 024201 (2017).

[43] Dirty Dirac semimetals with short-range $e$-e interaction have been studied by B. Roy and S. D. Sarma, Phys. Rev. B 94, 115137 (2016).

[44] $g_{\alpha \beta}$ represents the Minkowski metric with signature $g=$ $\operatorname{diag}(-1,1,1,1)$.

[45] See Supplemental Material at http://link.aps.org/supplemental/ 10.1103/PhysRevB.96.081104 for the details about the renormalization in the large- $N$ approach to interacting Weyl semimetals and the scaling of the critical disorder strength as a function of temperature in the noninteracting theory.

[46] D. J. Amit and V. Martín-Mayor, Field Theory, the Renormalization Group, and Critical Phenomena (World Scientific, Singapore, 2005).

[47] In our computation, the sum over frequencies is always cut off at a larger energy $\Lambda_{\omega} \gg v_{F} \Lambda$. 